\title{
Repository of mutations from Oman: The entry point to a
}

\section{national mutation database [version 1; peer review: 3}

\section{approved]}

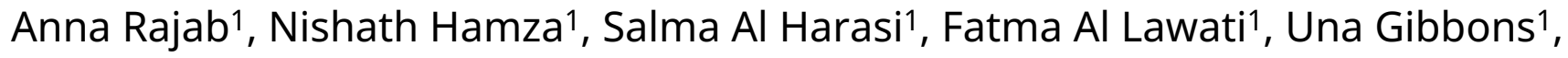 \\ Intesar Al Alawi ${ }^{1}$, Karoline Kobus ${ }^{1}$, Suha Hassan1, Ghariba Mahir², \\ Qasim Al Salmi², Barend Mons ${ }^{3}$, Peter Robinson ${ }^{4}$ \\ ${ }^{1}$ National Genetic Center, Ministry of Health, Muscat, PC 111, Oman \\ ${ }^{2}$ Royal Hospital, Ministry of Health, Muscat, PC 111, Oman \\ ${ }^{3}$ Bio-Semantics at the Department of Medical Informatics, Erasmus Medical Centre, University of Rotterdam, Rotterdam, 3015 CE, \\ The Netherlands \\ ${ }^{4}$ Institute of Medical Genetics and Human Genetics, Berlin, 13353, Germany
}

\begin{tabular}{l} 
V1 First published: 23 Sep 2015, 4:891 \\
https://doi.org/10.12688/f1000research.6938.1 \\
Latest published: 23 Sep 2015, 4:891 \\
https://doi.org/10.12688/f1000research.6938.1 \\
\hline
\end{tabular}

\begin{abstract}
The Sultanate of Oman is a rapidly developing Muslim country with well-organized government-funded health care services, and expanding medical genetic facilities. The preservation of tribal structures within the Omani population coupled with geographical isolation has produced unique patterns of rare mutations. In order to provide diagnosticians and researchers with access to an up-to-date resource that will assist them in their daily practice we collated and analyzed all of the Mendelian disease-associated mutations identified in the Omani population. By the $1^{\text {st }}$ of August 2015, the dataset contained 300 mutations detected in over 150 different genes. More than half of the data collected reflect novel genetic variations that were first described in the Omani population, and most disorders with known mutations are inherited in an autosomal recessive fashion. A number of novel Mendelian disease genes have been discovered in Omani nationals, and the corresponding mutations are included here. The current study provides a comprehensive resource of the mutations in the Omani population published in scientific literature or reported through service provision that will be useful for genetic care in Oman and will be a starting point for variation databases as nextgeneration sequencing technologies are introduced into genetic medicine in Oman.
\end{abstract}

\section{Keywords}

Genetic Disease, Birth Defects, disease-associated mutation data, Sultanate of Oman

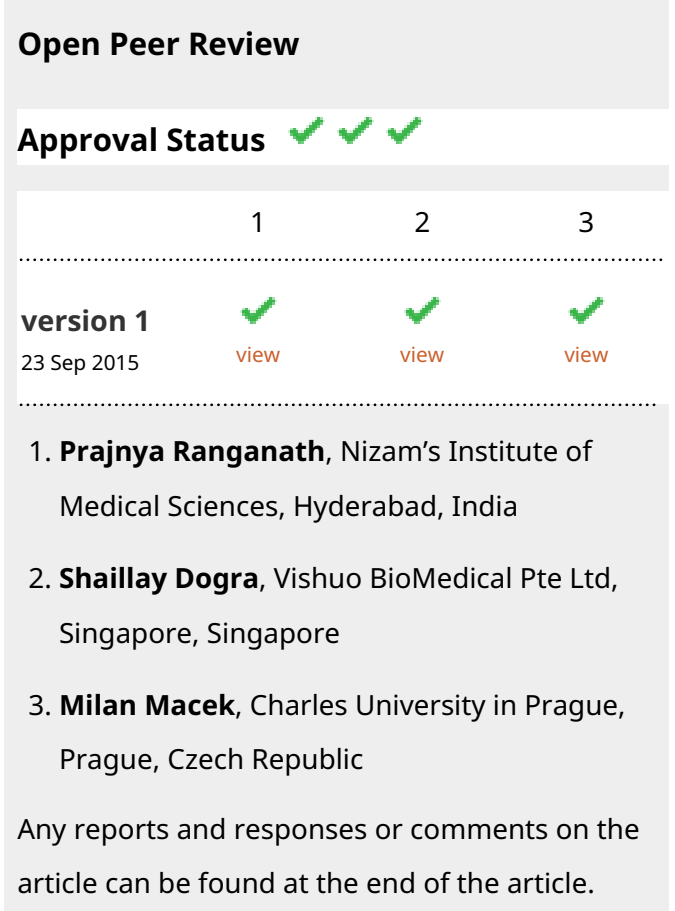


Corresponding author: Anna Rajab (drannarajab@gmail.com)

Competing interests: The authors declared no competing interests.

Grant information: The author(s) declared that no grants were involved in supporting this work.

Copyright: @ 2015 Rajab A et al. This is an open access article distributed under the terms of the Creative Commons Attribution License, which permits unrestricted use, distribution, and reproduction in any medium, provided the original work is properly cited.

How to cite this article: Rajab A, Hamza N, Al Harasi S et al. Repository of mutations from Oman: The entry point to a national mutation database [version 1; peer review: 3 approved] F1000Research 2015, 4:891 https://doi.org/10.12688/f1000research.6938.1

First published: 23 Sep 2015, 4:891 https://doi.org/10.12688/f1000research.6938.1 


\section{Introduction}

Oman is situated in the South East of the Arabian Peninsula along the East coast of the Arabian Gulf (Figure 1). It has its borders with United Arab Emirates to the North, Saudi Arabia to the West and Yemen to the South West. Oman is the second largest territory in the Arabian Peninsula with an area of 82,000 square miles and a coastline length of 1,300 miles. The native Omani population comprises around 2.2 million inhabitants, and the rate of annual population increase is approximately 25 per 1000 . Oman has a young population with nearly half of the population being under 15 years. The Omani population is characterized by a high growth rate, large family size, consanguineous marriages, and the presence of genetic isolates.
Clinical genetic services were introduced in the Sultanate of Oman in the past decade and they have become an important component of health care. This greatly facilitated the systematic collection of data on genetic diseases and birth defects in the past few decades. With the inauguration of the National Genetic Center in 2013, the existing clinical genetic services were supplemented by sophisticated genetic laboratory services.

The amount of published data available on genetic disorders in the Sultanate is considerable. There were a few previous attempts to list the genetic diseases reported in Oman ${ }^{1-4}$ and to link them to specific population groups and geographic locations ${ }^{5,6}$, analyze population

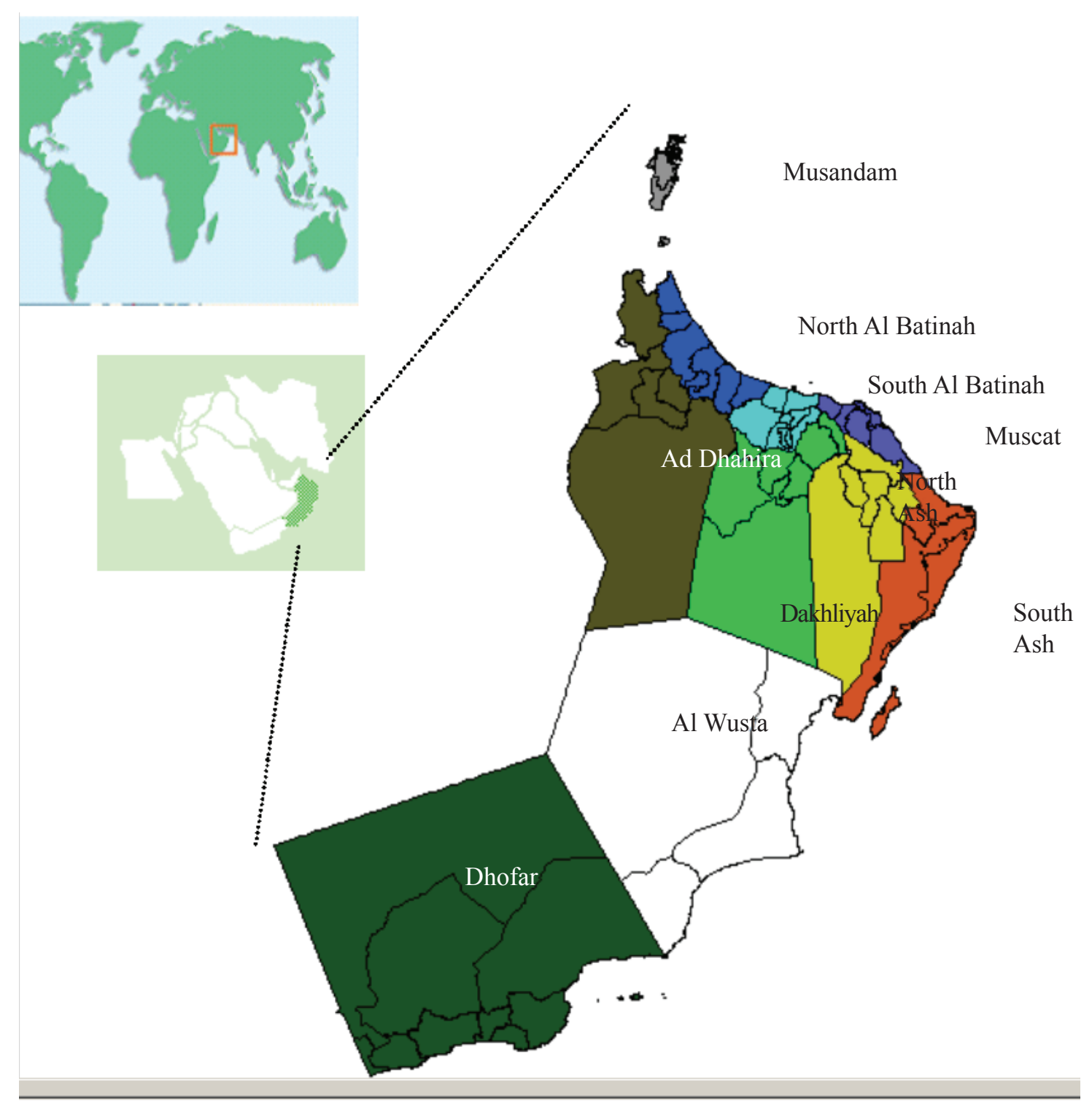

Figure 1. Oman is situated in the South East of the Arabian Peninsula along the East coast of the Arabian Gulf. 
structure $^{7}$, and to estimate the impact of genetic disorders and birth defects on the community ${ }^{4}$ and summarize the genetic services available ${ }^{8}$. The advances in bioinformatics required to annotate human genomic variants and to place them in public data repositories have not kept pace with their discovery. The deposition of such data in the public domain is essential to maximize both their scientific and clinical utility ${ }^{9}$.

Hence, in the current study we present a comprehensive compilation of germline mutations in nuclear genes associated with human disease in the Omani population.

\section{Materials and methods}

The wealth of genetic variant data in Omani nationals was collected from multiple sources which form a basis for research into genetic conditions reported from Oman. Multiple sources of data were reviewed to form repository of mutations in Omani nationals introduced in this paper. The sources of data included:

(1) 1993-2015 records of patients consulted by clinical geneticists of the Royal Hospital, the largest tertiary hospital in Oman;

(2) 2008-2015 publications curated from PUBMED on birth defects and genetic conditions in Omani nationals. The keywords used were: "Oman", "Genetic disorders", "Birth defects", "mutations";

(3) 2012-2014 commercial laboratories referral registry at the Royal Hospital for the samples tested overseas.

(4) The internal genetic variant repository of the National Genetic Center $<<$ HTTP://ogvd.net $>$;

The data presented in this article was manually curated. The OMIM identifiers, Phenotype MIM accession numbers, Phenotype name (OMIM), mutation descriptions, and relevant publications with PMID numbers were all collected from the NCBI database repository. All unavailable through PubMed mutation details were checked with ClinVar, LOVD and CentoMD. The details of unpublished mutations are not included in the present study and feature in Table 2 as "Novel mutations".

\section{Results}

In this study, a wide range of genetic conditions with known mutations collected in Omani nationals were analysed. The disease classifications are comprised of 44 gene variants causing neurodevelopmental disorders, 21 inborn errors of metabolism, 13 endocrinopathies, 15 skeletal dysplasias, nine disorders of the immune system, four hereditary blood disorders as well as other National groups (Table 1 ).

In total more than 150 rare genetic disorders were listed in Table 2 and Table 3 with relevant OMIM numbers, PubMed ID (PMID), Gene/Locus name, nucleotide(s) change(s) and the source of the data (PubMed ID Number/ OMIM/ClinVar/LOVD/CentoMD). The names of genetic conditions in Table 2 are stated as found in OMIM "Phenotype-Gene Relationships" table as "Phenotype" arranged in alphabetical order. In Table 3, we present a separate list of 69 known mutations (11-15) that were collected through service provision at the Hemoglobinopathy Laboratory at the National Genetic Center in Oman.

For the majority (85\%) of rare disorders presented in Table 2, data was derived from publications. The original mutations identified for the first time in Omani population constitute more than half of rare disease data presented in Table 2.

\section{Discussion}

Soon after the completion of the Human Genome Project in 2003 , it was clear that the genetic data collected until then presented only a glimpse of the complexity of the human genome and the significance of genetic variants in human disease. Since then, genetic researchers have unearthed innumerable variants that are not only individual-specific; but also ethnicity-, population- and country-specific. Human genetic variation databases have significant implications for both diagnostic and predictive medicine. Often, the pathogenicity of rare mutations is primarily assessed through multiple reports of occurrence in diseased patients that are documented and routinely updated in mutation databases. Given the fact that gene mutations and their frequencies in many Mendelian disorders differ widely between different ethnic groups, even within a country, national databases are highly valuable resources for studies on disease-gene associations, population diversity and genetic history ${ }^{10}$.

The catalog of Omani mutations presented here will therefore represent a valuable resource that may guide mutation analysis in Omanis suspected of having genetic disease. Unique circumstances in Oman with government-funded comprehensive healthcare throughout the country, and the national coverage for clinical genetics has made the present study possible. Future efforts will be required to extend this database to cover the full spectrum of mutations and population specific variants.

The disease-associated mutation data presented (Table 1, Table 2, Table 3) show a considerable proportion of novel disease genes as well as novel genetic variants within the Omani population. This was expected due to the presence of inbred and geographically isolated communities, the practice of consanguineous marriages, all of which have tended to skew the allelic spectrum toward rare and private variants within the Omani population. In addition to this, the list of genetic variants also reveals known mutations that were previously reported in certain non-Omani populations, thereby reflecting the historic genetic admixture that occurred in Oman, along the trade routes of a once powerful Omani empire and its foreign colonies. Many of the mutations reported are unique to the Omani population, suggesting a founder effect.

The interest in genetic testing is growing among physicians aiming to provide better medical care and genetic disease prevention. The data collected largely represent mutations of rare autosomal recessively inherited disorders in Oman. The mutation data in Table 2 can be searched by OMIM number, or by disease name. The names of diseases in Table 2 were chosen as described in OMIM in "Phenotype-Gene Relationships" table as "Phenotype" in order to ease finding specific genetic disorders by name. 
Table 1. Range of genetic conditions with known mutations in Omani nationals (details presented in Table 2 and Table 3 ).

\begin{tabular}{|c|c|c|c|c|}
\hline $\begin{array}{l}\text { Range of Genetic Conditions with } \\
\text { known mutations in Omani nationals }\end{array}$ & $\begin{array}{l}\text { Number } \\
\text { of disease } \\
\text { causing genes }\end{array}$ & $\begin{array}{l}\text { Number } \\
\text { of disease } \\
\text { causing } \\
\text { mutations }\end{array}$ & $\begin{array}{l}\text { Novel } \\
\text { Mutations of } \\
\text { known genes }\end{array}$ & $\begin{array}{l}\text { Novel genes } \\
\text { and novel } \\
\text { genetic } \\
\text { mechanisms }\end{array}$ \\
\hline \multicolumn{5}{|l|}{ Genetic Blood Disorders } \\
\hline Beta-Globin & 1 & 33 & 2 & 3 \\
\hline Alpha-Globin & 2 & 22 & 3 & 2 \\
\hline Delta-Globin & 1 & 14 & 3 & 0 \\
\hline Other hemolytic and hemorrhagic disorders & 3 & 5 & 3 & 2 \\
\hline \multicolumn{5}{|l|}{ Neurodevelopmental disorders } \\
\hline Conditions with intellectual disability & 20 & 23 & 9 & 7 \\
\hline Primary Microcephaly & 5 & 5 & 2 & 2 \\
\hline Epileptic syndromes & 2 & 2 & 0 & 1 \\
\hline Neurodegenerative conditions & 6 & 7 & 1 & 1 \\
\hline Syndromic ciliopathies & 6 & 7 & 5 & 3 \\
\hline Hereditary spastic paraplegias & 5 & 6 & 4 & 3 \\
\hline $\begin{array}{l}\text { Neuropathies and neuromuscular } \\
\text { disorders }\end{array}$ & 8 & 13 & 1 & 1 \\
\hline Arthrogryposis & 3 & 3 & 2 & 2 \\
\hline Inborn errors of metabolism & 21 & 28 & 21 & 2 \\
\hline Endocrine disorders & 13 & 28 & 2 & 5 \\
\hline Intrahepatic cholestasis and gut anomalies & 4 & 8 & 3 & 0 \\
\hline Disorders of the immune system & 9 & 15 & 3 & 4 \\
\hline Familial cancers & 4 & 6 & 1 & 0 \\
\hline Skeletal dysplasias and osteodysplasias & 15 & 23 & 6 & 8 \\
\hline Cardiogenetic conditions & 2 & 2 & 1 & 1 \\
\hline Renal disorders and dysplasias & 6 & 13 & 0 & 1 \\
\hline Skin, nails, and hair disorders & 6 & 10 & 0 & 3 \\
\hline Cutis laxa syndromes & 2 & 7 & 0 & 3 \\
\hline $\begin{array}{l}\text { Ophthalmological diseases including } \\
\text { blindness }\end{array}$ & 5 & 8 & 0 & 2 \\
\hline Congenital deafness & 1 & 3 & 0 & 1 \\
\hline Congenital lipodystrophies & 5 & 5 & 0 & 1 \\
\hline Cystic fibrosis & 1 & 4 & 1 & 2 \\
\hline Total & 156 & 300 & 83 & 58 \\
\hline
\end{tabular}

Extensive genetic studies were performed in Oman for Genetic Blood Disorders and various conditions leading to intellectual disabilities, mental and physical handicap. 
Table 2. List of disease-associated mutations in Omani nationals.

\begin{tabular}{|c|c|c|c|c|c|c|}
\hline No. & Phenotype (OMIM) & OMIM & PMIM ID & Gene/Locus & Nucleotide change & $\begin{array}{l}\text { Source: } \\
\text { Pubmed ID Nos/ } \\
\text { Registration at } \\
\text { international } \\
\text { databases }\end{array}$ \\
\hline \multirow{2}{*}{1} & \multirow{2}{*}{ Achondroplasia } & \multirow{2}{*}{100800} & \multirow{2}{*}{134934} & \multirow{2}{*}{ FGFR3 } & c.749G>C; & \multirow{2}{*}{ LOVD } \\
\hline & & & & & c. $1172 \mathrm{C}>\mathrm{A}$ & \\
\hline \multirow{5}{*}{2} & \multirow{5}{*}{$\begin{array}{l}\text { Adrenal hyperplasia, congenital, due } \\
\text { to } 21 \text {-hydroxylase deficiency }\end{array}$} & \multirow{5}{*}{201910} & \multirow{5}{*}{613815} & \multirow{5}{*}{ CYP 21A2 } & p. $1236 \mathrm{~N}$ & \multirow{5}{*}{21274396} \\
\hline & & & & & p.V237E & \\
\hline & & & & & p.M239K & \\
\hline & & & & & c. $306 \mathrm{~T}$ insert, & \\
\hline & & & & & $\begin{array}{l}\text { p.Q318X; conv Cyp 21P } \\
\text { to Cyp 21A2 }\end{array}$ & \\
\hline 3 & $\begin{array}{l}\text { Adrenal hyperplasia, congenital, due } \\
\text { to } 17 \text {-hydroxylase deficiency }\end{array}$ & 202110 & 609300 & CYP17A1 & $\begin{array}{l}\text { c. } 287 G>A \\
\text { p.Arg96Gln }\end{array}$ & 24498484 \\
\hline \multirow{2}{*}{4} & \multirow{2}{*}{ Allopecia universalis congenita } & \multirow{2}{*}{ *203655 } & \multirow{2}{*}{602302} & \multirow{2}{*}{$\mathrm{HR}$} & c. $2776+1, G>A$ & \multirow{2}{*}{9736769} \\
\hline & & & & & c. $1022 T>A$ & \\
\hline \multirow{2}{*}{5} & \multirow{2}{*}{ Alport syndrome, autosomal dominant } & \multirow{2}{*}{104199} & \multirow{2}{*}{104200} & \multirow{2}{*}{ COL4A3 } & c. $479 \mathrm{G}>\mathrm{A}$ & \multirow{2}{*}{14871398} \\
\hline & & & & & c.232delG & \\
\hline 6 & Alport syndrome, autosomal recessive & 203780 & 120070 & COL4A3 & $\mathrm{R} 1215 \times(\mathrm{CGA}>\mathrm{TG}$ & 14582039 \\
\hline \multirow[b]{2}{*}{7} & \multirow[b]{2}{*}{ Alport syndrome, X-linked (ATS) } & \multirow[b]{2}{*}{301050} & \multirow[b]{2}{*}{303630} & & $\begin{array}{l}\text { del exons } 7-8 \text { and } \\
31-36\end{array}$ & \\
\hline & & & & COL 4A5 & $\begin{array}{l}\text { potential duplication } \\
\text { of exons } 21-30+/- \\
\text { exon } 20\end{array}$ & 15149316 \\
\hline 8 & Alstrom syndrome & *606884 & 203800 & ALMS1 & Novel mutation & 17594715 \\
\hline & & & & & c.978T-to-ter & \\
\hline 9 & Amelogenesis imperfecta, type IIA3 & *613211 & 613214 & WDR72 & (V968X); & 19853237 \\
\hline & & & & & 2857 delA & \\
\hline & & & & & Exon 1: R74G ; & \\
\hline & & & & & Pdelta1nt & \\
\hline 10 & Apparent Mineralocorticoid & *0180? 0 & 611020 & HCR11R? & (P75Delta1nt) & 15121012 \\
\hline & ketoreductase deficiency & 210000 & 0itcuc & 年 & Exon2: L114Delta6nt & 年 \\
\hline & & & & & Exon 5: V322ins9nt & \\
\hline & & & & & Exon 3 :A221V & \\
\hline 11 & Arterial tortuosity syndrome & 208050 & 208050 & SLC2A10 & $243 C-G$ & 16550171 \\
\hline 12 & $\begin{array}{l}\text { Arthrogryposis, renal dysfunction, and } \\
\text { cholestasis } 1\end{array}$ & *608552 & 208085 & Vps33B & c.350delC & 15052268 \\
\hline 13 & $\begin{array}{l}\text { Autoimmune Lymphoproliferative } \\
\text { Syndrome (Type A) }\end{array}$ & 601859 & 601853 & FAS & c.232 del G, exon3 & 8787672 \\
\hline 14 & Bardet-Biedl Syndrome 9 (BBS9) & *615986 & 615986 & PTHB1 & IVS 17/IGTA variant & 17106446 \\
\hline 15 & Rardet-Riedl Syndrome 10 (RRS10) & *2099900 & 209900 & $\mathrm{Fl} 123560$ & n. $364 f s X 368$ & 17106446 \\
\hline J & 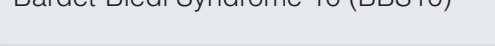 & 200000 & 200000 & 1 Luç & FLJ 23560 & 11 $1004+40$ \\
\hline 16 & $\begin{array}{l}\text { Brain Calcifications/Coat's like } \\
\text { syndrome/Rajab syndrome }\end{array}$ & *613658 & 613658 & NA & Linkage to $2 q 36.3$ & 19161147 \\
\hline 17 & Breast cancer & 114480 & 114480 & BRCA 1 & 2080insA & 18340530 \\
\hline 18 & $\begin{array}{l}\text { Carbamoylphosphate synthetase I } \\
\text { deficiency }\end{array}$ & *238970 & 237300 & CPSI & c.1590dupT & 22106832 \\
\hline
\end{tabular}




\begin{tabular}{|c|c|c|c|c|c|c|}
\hline No. & Phenotype (OMIM) & OMIM & PMIM ID & Gene/Locus & Nucleotide change & $\begin{array}{l}\text { Source: } \\
\text { Pubmed ID Nos/ } \\
\text { Registration at } \\
\text { international } \\
\text { databases }\end{array}$ \\
\hline 19 & $\begin{array}{l}\text { Carnitinepalmitoyl carboxylase } \\
\text { deficiency }\end{array}$ & *600650 & $1 \mathrm{p} 32.3$ & CPT2 gene & $\begin{array}{l}\text { detectable mutations } \\
\text { were excluded }\end{array}$ & ClinVar \\
\hline \multirow{2}{*}{20} & \multirow{2}{*}{$\begin{array}{l}\text { Central hypoventilation syndrome, } \\
\text { congenital }\end{array}$} & \multirow{2}{*}{603851} & \multirow{2}{*}{209880} & \multirow{2}{*}{ PHOX2B } & 5 Alanine Expansions & \multirow{2}{*}{ ClinVar } \\
\hline & & & & & 10 Alanine expansions & \\
\hline 21 & $\begin{array}{l}\text { Ceroid lipofuscinosis, neuronal, } \\
2 \text { (CLN2) }\end{array}$ & *204500 & 607998 & TPP1 & positive linkage & 17690061 \\
\hline 22 & Charcot-Marie-Tooth disease, type 4A & 214400 & 214400 & GDAP1 & Start-codon mutation & 22200116 \\
\hline 23 & Cohen Syndrome $(\mathrm{COH} 1)$ & *216550 & 216550 & VPS13B & $7934 G>A$ & 15173253 \\
\hline \multirow{4}{*}{24} & \multirow{4}{*}{ Cystic Fibrosis } & \multirow{4}{*}{ *219700 } & \multirow{4}{*}{$7 q 31.2$} & \multirow{4}{*}{ CFTR } & $102 T>A+S 549 R(T>G)]$ & \multirow{4}{*}{$\begin{array}{l}25829996 \\
10480369\end{array}$} \\
\hline & & & & & delta F508 & \\
\hline & & & & & F208Del; & \\
\hline & & & & & S549Rdel & \\
\hline 25 & Cholestasis intrahepatic & *243300 & 243300 & ATP8B1 & (exon 15) het del & $\begin{array}{l}\text { CentoMD; } \\
15239083\end{array}$ \\
\hline \multirow{2}{*}{26} & \multirow{2}{*}{$\begin{array}{l}\text { Cholestasis, benign recurrent } \\
\text { intrahepatic, } 2\end{array}$} & \multirow{2}{*}{605479} & \multirow{2}{*}{605479} & \multirow{2}{*}{ ABCB11 } & C. $149 G . A$ & \multirow{2}{*}{$\begin{array}{l}\text { LOVD; } \\
\text { CentoMD }\end{array}$} \\
\hline & & & & & c. $1416 \mathrm{~T} . \mathrm{A}$ & \\
\hline 27 & $\begin{array}{l}\text { Cholestasis, progressive familial } \\
\text { intrahepatic } 3\end{array}$ & 602347 & 602347 & ABCB4 & c. $2800 \mathrm{G}>\mathrm{A}$ & LOVD \\
\hline \multirow{3}{*}{28} & \multirow{3}{*}{$\begin{array}{l}\text { Cholestasis, intrahepatic, of } \\
\text { pregnancy, } 1\end{array}$} & \multirow{3}{*}{147480} & \multirow{3}{*}{147480} & \multirow{3}{*}{ ATP8B1 } & c. $1286 A>C$ & \multirow{3}{*}{$\begin{array}{l}\text { LOVD; } \\
\text { CentoMD }\end{array}$} \\
\hline & & & & & Novel mutation & \\
\hline & & & & & Novel mutation & \\
\hline \multirow{2}{*}{29} & \multirow{2}{*}{$\begin{array}{l}\text { Chondrodysplasia, Grebe type (Grebe } \\
\text { Acromesomelic Dysplasia) }\end{array}$} & \multirow{2}{*}{ *200700 } & 200700 & GDE5 & Del G1144; & 16636240 \\
\hline & & & & GDF5 & Transition A1137G & $10030<40$ \\
\hline 30 & Chronic granulomatous disease due & *233700 & 233700 & NCF1 & c. $579 \mathrm{G}>\mathrm{A}$ & 24446915; \\
\hline & todeficiency of NCF1 & 230100 & 230100 & TNCF & Novel mutation & 24446915 \\
\hline 31 & Chronic Granulomatous Disease, & *306400 & 306400 & CYBB, XK & $\begin{array}{l}\text { Del gp91-phox gene } \\
\text { (CYBB) }\end{array}$ & 24446915 \\
\hline & & & & & del McLeod gene (XK) & \\
\hline & Crialor Nai or Cundrome tyno 1 & & & & c. $211 \mathrm{G}>\mathrm{A}$ & \\
\hline 32 & Crigler-Naj ar Syndrome, type $\|$ & $606 / 85$ & $606 / 85$ & UGI1A1 & c. $1456 \mathrm{~T}>\mathrm{G}$ & 9630669 \\
\hline & Cutis laxa, autosomal recessive, & & & & c. $294+1 G 4 A$ & \\
\hline 33 & $\begin{array}{l}\text { type IIA (with congenital defect of } \\
\text { glycosulation) }\end{array}$ & *219200 & 219200 & ATP6V0A2 & c.1929delA & 18157129 \\
\hline & & & & & $356 G>A$ & \\
\hline & & & & & $566 \mathrm{C}>\mathrm{T}$ & \\
\hline 34 & $\begin{array}{l}\text { Cutis laxa, autosomal recessive, } \\
\text { type IIIB }\end{array}$ & *614438 & 614438 & PYCR1 & $356 G 4 A$ & 19648921 \\
\hline & & & & & c. $356 \mathrm{G}>\mathrm{A}$ & \\
\hline & & & & & c. $566 \mathrm{C}>\mathrm{T}$ & \\
\hline & & & & & S86T & 11748849 \\
\hline 35 & Deafness, autosomal recessive $1 \mathrm{~A}$ & *220290 & 2220290 & GJB2 & 35delG & $11 / 48849$ \\
\hline & & & & & 167delT & \\
\hline 36 & Diabetes mellitus, permanent neonatal & 606176 & 606176 & ABCC8 & c. $4480 \mathrm{C}>\mathrm{T}$ & 9769320 \\
\hline
\end{tabular}




\begin{tabular}{|c|c|c|c|c|c|c|}
\hline No. & Phenotype (OMIM) & OMIM & PMIM ID & Gene/Locus & Nucleotide change & $\begin{array}{l}\text { Source: } \\
\text { Pubmed ID Nos/ } \\
\text { Registration at } \\
\text { international } \\
\text { databases }\end{array}$ \\
\hline \multirow{7}{*}{37} & \multirow{7}{*}{ Dushenne Muscular Dystrophy } & \multirow{7}{*}{320200} & \multirow{7}{*}{320200} & \multirow{7}{*}{ DMD } & Del exon 7 & \multirow{7}{*}{19449031} \\
\hline & & & & & Dupl exons 55 to 77 & \\
\hline & & & & & c. $4996 C>T$ & \\
\hline & & & & & c.1733_1734delTA & \\
\hline & & & & & c. $1175 \mathrm{~T}>\mathrm{G}$ & \\
\hline & & & & & c. $1647 \mathrm{~T}>\mathrm{G}$ & \\
\hline & & & & & c.1521_1523delCTT & \\
\hline 38 & $\begin{array}{l}\text { Epiphyseal dysplasia, macrocephaly, } \\
\text { variable CC agenesis, spindle-shaped } \\
\text { fingers, mental retardation }\end{array}$ & *226900 & NA & $15 q 26$ & $\begin{array}{l}\text { Linkage D15S205/ } \\
\text { D15S966 }\end{array}$ & 11389160 \\
\hline 39 & $\begin{array}{l}\text { Ectodermal dysplasia 1, hypohidrotic, } \\
\text { X-linked (EDA) }\end{array}$ & 305100 & 305100 & ED1 & $\begin{array}{l}\text { c.G1113A; } \\
\text { Gly291Arg }\end{array}$ & 11279189 \\
\hline 40 & $\begin{array}{l}\text { Ectodermal dysplasia 10B, } \\
\text { hypohidrotic/hair/tooth type, } \\
\text { autosomal recessive }\end{array}$ & 224900 & 224900 & EDAR & 718delAAA & 20979233 \\
\hline 41 & $\begin{array}{l}\text { Enhanced S-cone syndrome (Golden- } \\
\text { Favre syndrome) }\end{array}$ & *268100 & 268100 & NR2E3 & C. $1117 \mathrm{~A}>\mathrm{G}$ & 24891813 \\
\hline \multirow{2}{*}{42} & \multirow{2}{*}{ Ellis Van-Creveld } & \multirow{2}{*}{ *225500 } & \multirow{2}{*}{225500} & \multirow{2}{*}{ EVC } & Frameshift in exon 13 & \multirow{2}{*}{$\begin{array}{l}17024374 \\
20184732\end{array}$} \\
\hline & & & & & Novel mutation & \\
\hline 43 & $\begin{array}{l}\text { Epilepsy, progressive myoclonic } 2 \mathrm{~B} \\
\text { (MELF) }\end{array}$ & 254780 & 254780 & NHLRC1 & c.468_469delAG & 18263761 \\
\hline 44 & Escobar syndrome & *265000 & 265000 & CHRNG & ү78dup(3) & 16826520 \\
\hline 45 & Ethyl Malonic Aciduria & 608451 & 608451 & ETHE1 & c. $487 \mathrm{C}>\mathrm{T}$ & $\begin{array}{l}\text { Cento MD; } \\
14732903\end{array}$ \\
\hline 46 & Factor $\mathrm{X}$ deficiency/Familiar CRM & *227600 & 227600 & F10 & c. $381 \mathrm{G}>\mathrm{A}$ & 12574802 \\
\hline \multirow{2}{*}{47} & \multirow{2}{*}{$\begin{array}{l}\text { Fanconi anemia, complementation } \\
\text { group D1 (FAD1) }\end{array}$} & \multirow{2}{*}{605724} & \multirow{2}{*}{605724} & \multirow{2}{*}{ BRCA2 } & $9609 C>T$ & \multirow{2}{*}{22660720} \\
\hline & & & & & exon 25 & \\
\hline \multirow{2}{*}{48} & \multirow{2}{*}{ Fanconi-Bickel Syndrome (GLUT2) } & \multirow{2}{*}{138160} & \multirow{2}{*}{227810} & \multirow{2}{*}{ SLC2A2 } & c. $1259 \mathrm{G}>\mathrm{T}$ & \multirow{2}{*}{22660720} \\
\hline & & & & & c. $1127 \mathrm{~T}>\mathrm{G}$ & \\
\hline 49 & Familial Mediterranean fever, AR & *608107 & 247100 & MEFV & c. $442 \mathrm{G}>\mathrm{C}$ & $\begin{array}{l}\text { CentoMD, } \\
\text { ClinVar }\end{array}$ \\
\hline \multirow{4}{*}{50} & \multirow{4}{*}{ Favism } & \multirow{4}{*}{305900} & \multirow{4}{*}{134700} & & c. $335 \mathrm{~A}>\mathrm{T}$ & \\
\hline & & & & G6PR & G6PD Chatham & 8860013 \\
\hline & & & & GorD & G6PD A- & . \\
\hline & & & & & 2 novel mutations & \\
\hline 51 & $\begin{array}{l}\text { Gastrointestinal defects and } \\
\text { immunodeficiency syndrome }\end{array}$ & 243150 & 243150 & TTC7A & Q712X & 25534311 \\
\hline 52 & Geroderma Osteodysplastica & *231070 & 231070 & SCYI 1RP1 & $\mathrm{C}-1 \_1: \mathrm{GA}>\mathrm{CT}$ & 18997784 \\
\hline & Hereditaria & & & 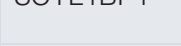 & 257delC & ד \\
\hline 53 & $\begin{array}{l}\text { Geroderma osteodysplastica } \\
\text { Hereditatria }\end{array}$ & *231070 & 231070 & GORAB & 367G-T & 19648921 \\
\hline & & & & & p.G61E & \\
\hline 54 & Glaucoma $3 \mathrm{~A}$, primary open angle, & *231300 & 231300 & CYP1R1 & p.D374N & 1959767 \\
\hline 34 & congenital, juvenile, or adult onset & 201000 & 231000 & ITID & p.R368H & 1909101 \\
\hline & & & & & p.E229K & \\
\hline
\end{tabular}




\begin{tabular}{|c|c|c|c|c|c|c|}
\hline No. & Phenotype (OMIM) & OMIM & PMIM ID & Gene/Locus & Nucleotide change & $\begin{array}{l}\text { Source: } \\
\text { Pubmed ID Nos/ } \\
\text { Registration at } \\
\text { international } \\
\text { databases }\end{array}$ \\
\hline \multirow{2}{*}{55} & \multirow{2}{*}{$\begin{array}{l}\text { Glycogen Storage Disease II, ACID } \\
\text { ALPHA-GLUCOSIDASE DEFICIENCY }\end{array}$} & \multirow{2}{*}{232300} & \multirow{2}{*}{232300} & \multirow{2}{*}{ GAA } & c. $2560 \mathrm{C}>\mathrm{T}$ & \multirow{2}{*}{ ClinVar } \\
\hline & & & & & c. $2105 \mathrm{G}>\mathrm{C}$ & \\
\hline 56 & Griscelli syndrome, type 2 & 607624 & 607624 & RAB27A & Novel mutation & NA \\
\hline 57 & $\begin{array}{l}\text { Hemolytic uremic syndrome, atypical, } \\
\text { susceptibility to, } 3\end{array}$ & 612923 & 612923 & $\mathrm{CFI}$ & c. $1332 A>G$ & CentoMD \\
\hline 58 & Hermansky-Pudlak syndrome 2 & 608233 & 608233 & AP3B1 & c.12_13delTA & $\begin{array}{l}16537806 \\
8042664\end{array}$ \\
\hline 59 & Hyperexplexia & *149400 & 149400 & GLRA1 & c. $593 \mathrm{G}>\mathrm{C}$ & 22264702 \\
\hline \multirow{6}{*}{60} & \multirow{6}{*}{$\begin{array}{l}\text { Hemophagocytic lymphohistiocytosis, } \\
\text { familial, } 2\end{array}$} & \multirow{6}{*}{ *603553 } & \multirow{6}{*}{603553} & \multirow{6}{*}{ PRF1 } & c. $265 \mathrm{C}>\mathrm{A}$ & \multirow{6}{*}{17674359} \\
\hline & & & & & c. 50delT & \\
\hline & & & & & c. $265>A$ & \\
\hline & & & & & .c. $674 \mathrm{G}>\mathrm{C}$ & \\
\hline & & & & & c.Del12bP & \\
\hline & & & & & $\begin{array}{l}\text { C. } 1122 \mathrm{G}>\mathrm{A} / \\
\text { Del12bP }\end{array}$ & \\
\hline \multirow{5}{*}{61} & \multirow{5}{*}{$\begin{array}{l}\text { Hyperinsulinemic hypoglycemia, } \\
\text { familial, } 1\end{array}$} & \multirow{5}{*}{ *256450 } & \multirow{5}{*}{256450} & \multirow{5}{*}{ ABCC8 } & c. $4480 \mathrm{C}>\mathrm{T}$ & \multirow{5}{*}{$\begin{array}{l}9769320 \\
25972930\end{array}$} \\
\hline & & & & & c. $.96 \mathrm{C}>\mathrm{A}$ & \\
\hline & & & & & c. $563 \mathrm{~A}>\mathrm{G}$ & \\
\hline & & & & & c. $119 \mathrm{~T}>\mathrm{G}$ & \\
\hline & & & & & 3 novel mutations & \\
\hline 62 & $\begin{array}{l}\text { Hyperinsulinemic Hypoglycemia, } \\
\text { familiar, } 5\end{array}$ & *147670 & 609968 & INSR & Novel mutation & NA \\
\hline 63 & Hypercholesterolemia, familial & *143890 & 143890 & LDLR & c.272delG & $\begin{array}{l}23162007 \\
24249837\end{array}$ \\
\hline 64 & $\begin{array}{l}\text { Hyperlipoproteinemia, type 1D } \\
\text { (chylomicronemia) }\end{array}$ & *615947 & 615947 & GPIHBP1 & C. $149 \mathrm{G}>\mathrm{A}$ & 22106832 \\
\hline 65 & $\begin{array}{l}\text { HYPERPHENYLALANINEM IA BH4- } \\
\text { deficient C, (HPABH4C) }\end{array}$ & *261630 & 261630 & QDPR & Novel mutation & NA \\
\hline 66 & Hyperoxaluria, primary, type 1 & 259900 & 259900 & AGXT & c.33-34insC & $\begin{array}{l}\text { CentoMD; } \\
21612638\end{array}$ \\
\hline 67 & $\begin{array}{l}\text { Homocystinuria due to MTHFR } \\
\text { deficiency }\end{array}$ & *236200 & 236250 & MTHFR & het $677 \mathrm{C}-\mathrm{T}$ & 15053809 \\
\hline 68 & $\begin{array}{l}\text { Hypoparathyroidism-retardation- } \\
\text { dysmorphism syndrome (Sanhad- } \\
\text { Sakati S) }\end{array}$ & 241410 & 241410 & TBCE & c. 155-166del12bp & 19491227 \\
\hline 69 & Hypophosphatasia, childhood & 241510 & 241510 & ALPL & c. $98 \mathrm{C}>\mathrm{T}$ & 25023282 \\
\hline 70 & Huntington Disease & 143100 & 143100 & $\mathrm{HTT}$ & $41-54$ repeats & 25689972 \\
\hline 71 & HUNTINTON-LIKE DISEASE & *605613 & NIL & HIP1R & Novel mutation & NA \\
\hline \multirow{2}{*}{72} & \multirow{2}{*}{$\begin{array}{l}\text { Ichthyosis, congenital, autosomal } \\
\text { recessive } 1\end{array}$} & \multirow{2}{*}{ *242300 } & \multirow{2}{*}{242300} & \multirow{2}{*}{ TGM1 } & c. $278 \mathrm{G}>\mathrm{A}$ & \multirow{2}{*}{23689228} \\
\hline & & & & & c. $396 \mathrm{~A}>\mathrm{H}$ & \\
\hline 73 & $\begin{array}{l}\text { Insensivity to pain, congenital, with } \\
\text { anhydrosis (HSAN IV) }\end{array}$ & 256800 & 256800 & NTRK1 & Novel mutation & NA \\
\hline \multirow{4}{*}{74} & \multirow{4}{*}{ Isovaleric acidemia } & \multirow{4}{*}{ *243500 } & \multirow{4}{*}{243500} & & p.F382fs; & \\
\hline & & & & IV & p.R392H; & 22960500 \\
\hline & & & & & p.R395Q; & 22960500 \\
\hline & & & & & p.E408K & \\
\hline
\end{tabular}




\begin{tabular}{|c|c|c|c|c|c|c|}
\hline No. & Phenotype (OMIM) & OMIM & PMIM ID & Gene/Locus & Nucleotide change & $\begin{array}{l}\text { Source: } \\
\text { Pubmed ID Nos/ } \\
\text { Registration at } \\
\text { international } \\
\text { databases }\end{array}$ \\
\hline 75 & Jouber Syndrome 1 (JBTS1) & *213300 & 213300 & INPPSE & c. $1546 \mathrm{C}>\mathrm{T}$ in exon 7 & 19668216 \\
\hline 76 & Joubert syndrome 5 & *610142 & 610188 & CEP 290 & c. $21 \mathrm{G}>\mathrm{T}$ exone1 & 19764032 \\
\hline 77 & Kindler Syndrome (poikiloderma) & *173650 & 173650 & KIND1 & $\mathrm{R} 271 \mathrm{X}$ & 12789646 \\
\hline 78 & Leprechaunism & 147670 & 246200 & INSR & $\begin{array}{l}\text { Single nucleotide del } \\
\text { in exon } 10\end{array}$ & $\begin{array}{l}\text { OMIM: } \\
\text { 147670.0028 }\end{array}$ \\
\hline 79 & $\begin{array}{l}\text { Leukodystrophy, hypomyelinating, } 2 \\
\text { (Pelizaeus-Merzbacher-Like Disease 1) }\end{array}$ & *608803 & 608804 & GJC2 & C. $-20+1 G>C$ & 23143715 \\
\hline 80 & LCHAD deficiency & 600980 & 609016 & HADHA & Novel mutation & NA \\
\hline C & $\begin{array}{l}\text { Lipodystrophy, congenital } \\
\text { generalized, type } 4\end{array}$ & *613327 & 613327 & PTRF-Cavin & c. $160 \mathrm{delG}$ & 20300641 \\
\hline 82 & $\begin{array}{l}\text { Lipodystrophy, congenital } \\
\text { generalized, type } 1 \text { (BSCL1) }\end{array}$ & 608594 & 608594 & AGPAT2 & $\begin{array}{l}\text { Homozygosity } \\
\text { D9S1818-DS1826 }\end{array}$ & 11916958 \\
\hline 83 & $\begin{array}{l}\text { Lipodystrophy, congenital } \\
\text { generalized, type } 2 \text { (BSCL2) }\end{array}$ & 606158 & 269700 & SEIPIN & $\begin{array}{l}\text { Homozygocity } 1883- \\
4136\end{array}$ & 12116229, \\
\hline 84 & Lipodystrophy, familial partial, 2 & 150330 & 151660 & LAMIN AIC & Homozygosity 3757 & 12116229 \\
\hline 85 & $\begin{array}{l}\text { Limb Girdle muscular dystrophy } \\
\text { 2B;LGMD2B (Miyoshi myopathy) }\end{array}$ & 254130 & 254130 & DYSF & $C: 526 C>T$ & 10469840 \\
\hline 86 & Lissencephaly LIS 4A & 300121 & 300121 & DCX & exon 5: & 11175293 \\
\hline 87 & Loeys-Dietz syndrome, type 1 & 609192 & 609192 & TGFBR1 or 2 & Positive linkage & 16928994 \\
\hline 88 & Long QT syndrome 1(LQT1) & 192500 & 192500 & KCNQ1 & $1388 G>C$ & 15159330 \\
\hline \multirow{2}{*}{89} & \multirow{2}{*}{ MODY type II), Glucokinase related } & \multirow{2}{*}{ *125851 } & \multirow{2}{*}{125891} & \multirow{2}{*}{ GCK } & c. $757 \mathrm{G}>\mathrm{T}$ & \multirow{2}{*}{24993573} \\
\hline & & & & & c. $292 \mathrm{C}>\mathrm{T}$; & \\
\hline 90 & $\begin{array}{l}\text { Mental retardation, autosomal } \\
\text { recessive } 43 \text { (MRT 43) }\end{array}$ & *615817 & 615817 & KIAA1033 & $\begin{array}{l}\text { c.3056C-G } \\
\text { transversion in exon } 29\end{array}$ & 2149877 \\
\hline 91 & $\begin{array}{l}\text { Mental Retardation, autosomal } \\
\text { recessive }\end{array}$ & *602810 & 602810 & HIST 3H3 & c. R130C & 21937992 \\
\hline 92 & $\begin{array}{l}\text { Mental Retardation Autosomal } \\
\text { Recessive, epilepsy, autism }\end{array}$ & *NA & NA & DEAF1 & c. $997+4 A>C$ & 26048982 \\
\hline 93 & Meckel Gruber syndrome (MKS 3) & *607361 & 607361 & TMEM67 & c. $383-384 \mathrm{AC}$ del & 16415887 \\
\hline 94 & $\begin{array}{l}\text { Microcephaly with simplified gyral } \\
\text { pattern }\end{array}$ & *603807 & 603807 & NA & Excluded known loci & 17975804 \\
\hline 95 & $\begin{array}{l}\text { Microcephaly } 3 \text {, primary, autosomal } \\
\text { recessive }\end{array}$ & 604804 & 604804 & CDK5RAP2 & c. E234X & 22887808 \\
\hline 96 & $\begin{array}{l}\text { Microcephaly 5, primary, autosomal } \\
\text { recessive }\end{array}$ & 608716 & 608716 & ASPM & c.9153_9154 del ins A & 15045028 \\
\hline \multirow{2}{*}{97} & \multirow{2}{*}{ Microcephaly and hypomielination } & \multirow{2}{*}{$\begin{array}{l}\text { *Omim } \\
179035\end{array}$} & \multirow{2}{*}{ NA } & \multirow{2}{*}{ PYCR2 } & c. $355 \mathrm{C}>\mathrm{T}$ & \multirow{2}{*}{25865492} \\
\hline & & & & & c. $751 \mathrm{C}>\mathrm{T}$ & \\
\hline 98 & $\begin{array}{l}\text { Microcephalic osteodysplastic } \\
\text { primordial dwarfism, type II }\end{array}$ & *210720 & 210720 & PCNT & Maps to $21 \mathrm{q} 22.3$ & 18174396 \\
\hline \multirow[b]{2}{*}{99} & \multirow[b]{2}{*}{ Mucolipidosis IV } & \multirow[b]{2}{*}{252650} & \multirow[b]{2}{*}{252650} & \multirow[b]{2}{*}{ MCOLN1 } & c. $1207 \mathrm{C}>\mathrm{T}$ & \multirow{2}{*}{$\begin{array}{l}\text { 15523648; } \\
\text { 11030752; } \\
16287144\end{array}$} \\
\hline & & & & & $\begin{array}{l}\text { Het NM_020533: } \\
\text { c. } 1208 G>T \text {. }\end{array}$ & \\
\hline 100 & $\begin{array}{l}\text { Mucopolysaccharidosis type IVB } \\
\text { (Morquio) }\end{array}$ & 253010 & 252010 & GLB1 & C. $1420 \mathrm{G}>\mathrm{C}$ & CentoMD \\
\hline 101 & $\begin{array}{l}\text { Multiple endocrine neoplasia IIA } \\
\text { (MEN2A) }\end{array}$ & 171400 & 171400 & RET & c. $1900 T>C$ & 8103403 \\
\hline
\end{tabular}




\begin{tabular}{|c|c|c|c|c|c|c|}
\hline No. & Phenotype (OMIM) & OMIM & PMIM ID & Gene/Locus & Nucleotide change & $\begin{array}{l}\text { Source: } \\
\text { Pubmed ID Nos/ } \\
\text { Registration at } \\
\text { international } \\
\text { databases }\end{array}$ \\
\hline \multirow{2}{*}{102} & \multirow{2}{*}{ Menkes Disease (Kinky Hair Disease) } & \multirow{2}{*}{309400} & \multirow{2}{*}{309400} & ATP7A & \multirow{2}{*}{ Novel mutation } & \multirow{2}{*}{ CentoMD } \\
\hline & & & & Xq21.1 & & \\
\hline 103 & $\begin{array}{l}\text { Multiple pterygium syndrome, lethal } \\
\text { type }\end{array}$ & *100730 & 253290 & CHRNG & c.ARG448TER & 16826520 \\
\hline 104 & Myotonic Dystrophy 1 & 605377 & 160900 & DMPK & $\begin{array}{l}\text { Expansion, >rpts } \\
\text { 1allele }\end{array}$ & 8036515 \\
\hline \multirow{2}{*}{105} & \multirow{2}{*}{ Nephrotic syndrome, type 1} & \multirow{2}{*}{ *256300 } & \multirow{2}{*}{25630} & \multirow{2}{*}{ NPHS1 } & (121delCT) ; & \multirow{2}{*}{$\begin{array}{l}\text { CentoMD; } \\
9660941\end{array}$} \\
\hline & & & & & c. $218 \mathrm{C}>\mathrm{T}$ & \\
\hline \multirow{3}{*}{106} & \multirow{3}{*}{$\begin{array}{l}\text { Nephrotic syndrome, type 2, steroid } \\
\text { resistant (NPHS2; SRN1) }\end{array}$} & \multirow{3}{*}{ *600995 } & \multirow{3}{*}{600995} & \multirow{3}{*}{ NPHS2 } & c.467Dup/c.709G>; & \multirow{3}{*}{$\begin{array}{l}\text { CentoMD; } \\
15817495\end{array}$} \\
\hline & & & & & c. $709 \mathrm{G}>\mathrm{C}$ & \\
\hline & & & & & c.779T>A; & \\
\hline 107 & Noonan syndrome 1 (NS1) & 163150 & 163150 & PTPN11 & c. $218 \mathrm{C}>\mathrm{T}$ & 12161469 \\
\hline 108 & $\begin{array}{l}\text { Niemann-Pick disease, type C1 } \\
\text { (NPC1) }\end{array}$ & *257220 & 257220 & NPC1 & c. $3362 T>G$ & Cento MD \\
\hline \multirow{2}{*}{109} & \multirow{2}{*}{ Osteogenesis imperfecta, type VIII } & \multirow{2}{*}{610915} & \multirow{2}{*}{610915} & \multirow{2}{*}{ LEPRE1 } & c. $2075-1 \mathrm{G}>\mathrm{A}$ & \multirow{2}{*}{$\begin{array}{l}\text { 24498616; } \\
\text { LOVD }\end{array}$} \\
\hline & & & & & c. $1170+9 G>A$ & \\
\hline 110 & Osteogenesis imperfecta, type VI & 613982 & 613982 & SERPINF1 & c. $-9+2$ dup & 23054245 \\
\hline 111 & Osteopetrosis, infantilile malignant & *259700 & 259700 & TCIRG1 & c.-XY_-YZdel & 23685543 \\
\hline 112 & Orofaciodigital syndrome V & *174300 & 174300 & DDX59 & C. $1600 \mathrm{G}>\mathrm{A}$ & 23972372 \\
\hline \multirow{2}{*}{113} & \multirow{2}{*}{$\begin{array}{l}\text { Paroxysmal nonkinesigenic dyskinesia } \\
\text { (PNKD1) }\end{array}$} & \multirow{2}{*}{ *118800 } & \multirow{2}{*}{118800} & \multirow{2}{*}{ MR-1 gene } & c. $20 \mathrm{C}>\mathrm{T}: \mathrm{A} 7 \mathrm{~V}$ & 16632198 \\
\hline & & & & & c. $26 \mathrm{C}>\mathrm{T}: \mathrm{A9V})$ & 10032198 \\
\hline 114 & $\begin{array}{l}\text { Peroxisome biogenesis disorder } 1 \mathrm{~A} \\
\text { (Zellweger) }\end{array}$ & 602136 & 214100 & PEX-1 & $\begin{array}{l}\text { c. } 1927 \_1928 d u p A ; \text {. c. } \\
2088 A>G\end{array}$ & $\begin{array}{l}\text { PMCID: } \\
2649967 \\
25407003\end{array}$ \\
\hline 115 & Pelger-Huet anomaly & *169400 & 169400 & LBR & $\begin{array}{l}\text { del } 6 \text { BP in splice site } \\
\text { intron } 12\end{array}$ & 21326950 \\
\hline & & & & & c.771dup.A; & \\
\hline 116 & Pheochromosytoma/paraganglioma 4 & 115310 & 115310 & SDHB & c. $574 \mathrm{~T}>\mathrm{C}$ & $\begin{array}{l}25034258 \\
15328326\end{array}$ \\
\hline & & & & & c. $859 \mathrm{G}>\mathrm{A}$ & \\
\hline 117 & $\begin{array}{l}\text { Pituitary hormone deficiency, } \\
\text { combined, } 3\end{array}$ & *221750 & 221750 & LHX3 & 3,088-bp deletion & 18407919 \\
\hline 118 & Pontocerebellar Hypoplasia type III & *608027 & 608027 & PCLO & $\begin{array}{l}\text { nonsense mutation of } \\
P C L O \text { (piccolo) gene }\end{array}$ & 25832664 \\
\hline 119 & $\begin{array}{l}\text { Polycystic Kidney and Hepatic } \\
\text { Disease } 1\end{array}$ & 263200 & 263200 & FCYT & c. $107 \mathrm{C}>\mathrm{T}$ & 11919560 \\
\hline 120 & Polycystic liver disease & 608648 & 608648 & SEC63 & $\begin{array}{l}\text { Del in promoter } \\
\text { region }\end{array}$ & $\begin{array}{l}24886261 \\
25165181\end{array}$ \\
\hline 121 & Rabson-Mendenhall syndrome & 262190 & 262190 & INSR & c.671_685dup & $\begin{array}{l}\text { CentoMD; } \\
22824672\end{array}$ \\
\hline 122 & Rajab Syndrome & *613658 & 613658 & NA & $\begin{array}{l}\text { linkage D2S351/ } \\
\text { D2S2390 }\end{array}$ & 19161147 \\
\hline 123 & Renal tubular acidosis, distal, AR, with & *611590 & 611590 & SI C4A1 & A858D; & 22126643 \\
\hline & hemolitic anaemia & & & & A858D/N & \\
\hline 124 & $\begin{array}{l}\text { Retinitis pigmentosa-12, autosomal } \\
\text { recessive }\end{array}$ & *604210 & 600105 & RABS 1 & 7 mutations & 24512366 \\
\hline 125 & Retinitis pigmentosa 37 & *604485 & 268100 & NR2E3 & p.D406G & 24891813 \\
\hline
\end{tabular}




\begin{tabular}{|c|c|c|c|c|c|c|}
\hline No. & Phenotype (OMIM) & OMIM & PMIM ID & Gene/Locus & Nucleotide change & $\begin{array}{l}\text { Source: } \\
\text { Pubmed ID Nos/ } \\
\text { Registration at } \\
\text { international } \\
\text { databases }\end{array}$ \\
\hline 126 & Rett Syndrome & 312750 & 312750 & MECP2 & c. $880 \mathrm{C}>\mathrm{T}$ & ClinVar \\
\hline \multirow{2}{*}{127} & \multirow{2}{*}{$\begin{array}{l}\text { Robinow syndrome, autosomal } \\
\text { recessive }\end{array}$} & \multirow{2}{*}{ *268310 } & \multirow{2}{*}{268310} & \multirow{2}{*}{ ROR 2} & c. $1504 C>T$ & \multirow{2}{*}{$\begin{array}{l}\text { 10932186; } \\
19640924\end{array}$} \\
\hline & & & & & c. $1324 C>T$ & \\
\hline 128 & $\begin{array}{l}\text { Severe combined immunodeficiency, } \\
\text { B cell-negative }\end{array}$ & 179615 & 601457 & RAG1 & c. $1187 G>A$ & ClinVar \\
\hline \multirow{2}{*}{129} & \multirow{2}{*}{ Spinal Muscular Atrophy (SMN1) } & \multirow{2}{*}{253300} & \multirow{2}{*}{253300} & \multirow{2}{*}{ SMN1 } & del exons 5, 6, 8 ; & \multirow{2}{*}{$\begin{array}{l}15000810 \\
17940251\end{array}$} \\
\hline & & & & & Del 5q13.2 in exon 7 & \\
\hline 130 & $\begin{array}{l}\text { Spastic paraplegia 18, autosomal } \\
\text { recessive (IDMDC) }\end{array}$ & *611225 & 611225 & ERLIN 2 & $\begin{array}{l}\text { (D8S1820 and } \\
\text { D8S532) }\end{array}$ & 16636240 \\
\hline 131 & $\begin{array}{l}\text { Spastic paraplegia 20, SPG20; (Troyer } \\
\text { Syndrome) }\end{array}$ & *275900 & 275900 & SPG 20 & c. $123 \mathrm{X}$ & 20437587 \\
\hline 132 & $\begin{array}{l}\text { Spastic paraplegia 35, autosomal } \\
\text { recessive; (FAHN); Leukodystrophy, } \\
\text { dysmyelinating, and spastic } \\
\text { paraparesis }\end{array}$ & 612319 & 612319 & $\mathrm{FA} 2 \mathrm{H}$ & c. $235 \mathrm{~A}>\mathrm{C}$ & 20104589 \\
\hline 133 & $\begin{array}{l}\text { Spastic paraplegia 54, autosomal } \\
\text { recessive }\end{array}$ & *615033 & 615003.0005 & DDHD2 & 1546C-T transition & 23176823 \\
\hline 134 & $\begin{array}{l}\text { Spastic paraplegia, ataxia, and } \\
\text { mental retardation }\end{array}$ & *607565 & 607565 & GRID2 & Novel mutation & NA \\
\hline 135 & $\begin{array}{l}\text { Split-hand/foot malformation with long } \\
\text { bone deficiency } 3\end{array}$ & *612576 & 612576 & BHLHA9 & microduplications & 22147889 \\
\hline 136 & $\begin{array}{l}\text { Spondylometaepiphyseal dysplasia, } \\
\text { short limb-hand type (SMED-SL) }\end{array}$ & *271665 & 271665 & DDR2 & c.2468_2469del CT & 24725993 \\
\hline 137 & $\begin{array}{l}\text { Spondyloepiphyseal dysplasia Omani } \\
\text { type with congenital joint dislocations }\end{array}$ & *143095 & 1439095 & CHST3 & c. $911 \mathrm{G}>\mathrm{A}$ & 15215498 \\
\hline 138 & $\begin{array}{l}\text { Spondylocostal dysostosis } 2 \text {, } \\
\text { autosomal recessive }\end{array}$ & 605915 & 608681 & MESP2; & c. $880 \mathrm{C}>\mathrm{T}$ & ClinVar \\
\hline 139 & $\begin{array}{l}\text { Spinocerebellar ataxia 7; } \\
\text { Olivopontocerebellar atrophy III; } \\
\text { ADCA type II }\end{array}$ & 164500 & 164500 & ATXN7; & $\begin{array}{l}\text { Repeat expansion of } \\
\text { ATXN7 gene }\end{array}$ & ClinVar \\
\hline 140 & Schwartz-Jampel syndrome, type 1 & *255800 & 255800 & HSPG-2 & $\begin{array}{l}\text { IVS64DS, A-G, +4; } \\
\text { c. } 1532 \mathrm{C}>\mathrm{T}\end{array}$ & 11101850 \\
\hline \multirow{2}{*}{141} & \multirow{2}{*}{$\begin{array}{l}\text { Stuve-Wiedemann syndrome/ } \\
\text { Schwartz-Jampel type } 2 \text { syndrome }\end{array}$} & \multirow{2}{*}{601559} & \multirow{2}{*}{601559} & \multirow{2}{*}{ LIFR } & c.653_654insT & \multirow{2}{*}{14740318} \\
\hline & & & & & c.643del T; & \\
\hline 142 & $\begin{array}{l}\text { Systemic Lupus Erythematosus (SLE), } \\
\text { susceptibility. }\end{array}$ & *125505 & 152700 & DNASE1L3 & G38OR ; & 22019780 \\
\hline \multirow{2}{*}{143} & \multirow{2}{*}{ Thanatophoric Dysplasia type 1} & \multirow{2}{*}{ *187600 } & \multirow{2}{*}{187600} & \multirow{2}{*}{ FGFR-3 } & $\mathrm{R} 248 \mathrm{C}$ & \multirow{2}{*}{12633765} \\
\hline & & & & & c. $4406 A>G$ & \\
\hline \multirow{2}{*}{144} & \multirow{2}{*}{ Three-M syndrome 1} & \multirow{2}{*}{ *273750 } & \multirow{2}{*}{273750} & \multirow{2}{*}{ CUL } & $c 2434 \mathrm{C}>\mathrm{T}$ & 19225462 \\
\hline & & & & & 690 ins C & 19877176 \\
\hline 145 & Three M Syndrome 2 & *610991 & 612921 & OBCL1 & 844ins68 & 19877176 \\
\hline 146 & Thrombosis, hyperhomocysteinemic & *236200 & 236200 & CBS & c. $807 \mathrm{C}>\mathrm{A}$ & 16432849 \\
\hline \multirow{2}{*}{147} & \multirow{2}{*}{$\begin{array}{l}\text { Thyroid hormone resistance, } \\
\text { autosomal recessive }\end{array}$} & 190160 & 274300 & TRR? & del in exon10 & 1991834 \\
\hline & & 年 & 214000 & 1110ट & Novel mutation & NA \\
\hline 148 & von Hippel-Lindau syndrome (VHL) & *193300 & 193300 & VHL & Novel mutation & NA \\
\hline
\end{tabular}

The disorders are listed in alphabetical order along with the mutations detected in Omani patients. Novel genes and/or mutations identified for the first time in Omani nationals are marked by an asterisk $\left(^{*}\right)$. Unpublished mutation data referred as "Novel mutations" would be updated following publication, currently source stated as "NA". 
The number of collected mutations among different disease groups (Table 1) reflect the frequency of disorders in the Omani population, the burden caused by genetic diseases ${ }^{4}$, and the interests of individual clinicians in genetic testing.

The knowledge of the genetics of Hemoglobin disorders is among the best in Oman due to national preventive programs and research starting from the 1990s. It is not surprising that around a third of all mutations known in Omani population to date are in four genes causing Hemoglobin disorders (Table 1, Table 3). The birth prevalence of infants with a hemoglobin disorder was recorded as $3.5-4.7 / 1,000^{7,11}$. The frequency of hemoglobin disorders in Oman is among the highest in the world, and may reflect natural selection due to advantage for survival, in the heterozygous state, against

\begin{tabular}{|c|c|c|c|c|}
\hline Disease & OMIM & Gene/Locus & Nucleotide change & PMID \\
\hline \multirow{10}{*}{$\begin{array}{l}\text { Sickle cell anemia/ } \\
\text { Hemoglobin variants }\end{array}$} & \multirow{10}{*}{603903} & \multirow{10}{*}{ HBB } & c. $19 \mathrm{G}>\mathrm{A}$ & 25677748 \\
\hline & & & c. $20 \mathrm{~A}>\mathrm{T}$ & 81926 \\
\hline & & & c. $92 \mathrm{G}>\mathrm{C}$ & 25677748 \\
\hline & & & c. $97 \mathrm{C}>\mathrm{G}$ & 1517102 \\
\hline & & & c. $79 \mathrm{G}>\mathrm{A}$ & 25677748 \\
\hline & & & c. $176 \mathrm{C}>\mathrm{A}$ & 25677748 \\
\hline & & & c. $176 \mathrm{C}>\mathrm{G}$ & 25677748 \\
\hline & & & c. $364 \mathrm{G}>\mathrm{A}$ & 25677748 \\
\hline & & & c. $364 G>C$ & 25677748 \\
\hline & & & c. $389 \mathrm{C}>\mathrm{T}$ & 25677748 \\
\hline \multirow{23}{*}{ Beta Thalassemia } & \multirow{23}{*}{613985} & \multirow{23}{*}{ HBB } & c. $-151 \mathrm{C}>\mathrm{T}$ & 25677748 \\
\hline & & & c. $-138 \mathrm{C}>\mathrm{A}$ & 25677748 \\
\hline & & & c. $-121 \mathrm{C}>\mathrm{T}^{*}$ & 21801233 \\
\hline & & & c. $-102 G>T^{\star}$ & 25826385 \\
\hline & & & c.17_18delCT & 25677748 \\
\hline & & & c.27_28insG & 25677748 \\
\hline & & & c. $32 \mathrm{C}>\mathrm{T}$ & 25677748 \\
\hline & & & c. $47 \mathrm{G}>\mathrm{A}$ & 20353347 \\
\hline & & & c.51delC & 20353347 \\
\hline & & & c. $90 \mathrm{C}>\mathrm{T}$ & 25677748 \\
\hline & & & c. $92 \mathrm{G}>\mathrm{A}$ & 25677748 \\
\hline & & & c. $92+1 G>A$ & 25677748 \\
\hline & & & c. $92+5 G>C$ & 25677748 \\
\hline & & & c. $92+6 \mathrm{~T}>\mathrm{C}$ & 25677748 \\
\hline & & & c.93-22_95del & 25677748 \\
\hline & & & c. $93-21 \mathrm{G}>\mathrm{A}$ & 25677748 \\
\hline & & & c.93-3T>G & 25677748 \\
\hline & & & c. $118 \mathrm{C}>\mathrm{T}$ & 25677748 \\
\hline & & & c.126_129delCTTT & 20353347 \\
\hline & & & c. 135 delC & 25677748 \\
\hline & & & c. $315+1 G>A$ & 25677748 \\
\hline & & & c. $316-2 A>G$ & 25677748 \\
\hline & & & c. ${ }^{*} 108$ * 112 delAATAA & 25677748 \\
\hline
\end{tabular}




\begin{tabular}{|c|c|c|c|c|}
\hline Disease & OMIM & Gene/Locus & Nucleotide change & PMID \\
\hline \multirow{22}{*}{ Alpha Thalassemia } & \multirow{22}{*}{604131} & \multirow{22}{*}{ HBA1/HBA2 } & c. $24 \mathrm{G}>\mathrm{C}$ & 25370869 \\
\hline & & & c. $38 \mathrm{C}>\mathrm{A}$ & 25370869 \\
\hline & & & c. $43 \mathrm{~T}>\mathrm{C}$ & 25370869 \\
\hline & & & c. $55 \mathrm{G}>\mathrm{C}$ & 24165563 \\
\hline & & & c. $56 \mathrm{G}>\mathrm{A}$ & 25370869 \\
\hline & & & c.56delG & 25370869 \\
\hline & & & c. $64 \mathrm{G}>\mathrm{C}$ & 25370869 \\
\hline & & & c. $71 \mathrm{~A}>\mathrm{T}$ & 5675637 \\
\hline & & & c.95+2_c.95+6delTGAGG & 25370869 \\
\hline & & & C.118_120delACC & 25370869 \\
\hline & & & c. $181 A>G$ & 25370869 \\
\hline & & & c. $264 C>G$ & 25826385 \\
\hline & & & c.283_300+3dup* & 25370869 \\
\hline & & & c. $326 \mathrm{C}>\mathrm{A}$ & 16840225 \\
\hline & & & c. $427 \mathrm{~T}>\mathrm{C}$ & 25370869 \\
\hline & & & c. ${ }^{*} 92 A>G$ & 25370869 \\
\hline & & & c. ${ }^{*} 94 A>G$ & 25370869 \\
\hline & & & Hybrid $3.7-5(\mathrm{C}>\mathrm{T})^{\star}$ & 25370869 \\
\hline & & & Hybrid $3.7+46(C>A)^{*}$ & 25370869 \\
\hline & & & $\begin{array}{l}\text { - -(MED-I); deletion of } \sim 17.5 \mathrm{~kb} \text { including } \\
\text { both alpha-globin genes }\end{array}$ & 25370869 \\
\hline & & & $3.7 \mathrm{~kb}$ deletion & 25370869 \\
\hline & & & $4.2 \mathrm{~kb}$ deletion & 25370869 \\
\hline \multirow{14}{*}{ Delta Thalassemia } & \multirow{14}{*}{142000} & \multirow{14}{*}{$\mathrm{HBD}$} & c. $-118 \mathrm{C}>\mathrm{T}$ & 24985928 \\
\hline & & & c. $14 \mathrm{C}>\mathrm{T}$ & 24985928 \\
\hline & & & c. $49 \mathrm{G}>\mathrm{C}$ & 24985928 \\
\hline & & & c. $68 \mathrm{C}>\mathrm{A}$ & 6058951 \\
\hline & & & c. $82 \mathrm{G}>\mathrm{T}$ & 24985928 \\
\hline & & & c. $93-1 G>C$ & 24985928 \\
\hline & & & c. $295 \mathrm{G}>\mathrm{A}$ & 2477064 \\
\hline & & & c. $301 C>T$ & 24985928 \\
\hline & & & c.333-334insGT* & 24985928 \\
\hline & & & c. $350 \mathrm{G}>\mathrm{A}$ & 24985928 \\
\hline & & & c. $410 \mathrm{G}>\mathrm{A}$ & 24985938 \\
\hline & & & c. $422 \mathrm{C}>\mathrm{T}$ & 17145605 \\
\hline & & & c. $427 G>C^{*}$ & 25043855 \\
\hline & & & c. $443 G>T^{*}$ & 24985928 \\
\hline
\end{tabular}

The different mutations reported by the National Genetic Center in patients with Hemoglobinopathies in Oman. Novel mutations are indicated by an asterisk $\left(^{*}\right)$ indicated to the left side of the mutation. Mutations are listed in ascending order based on nucleotide position. 
malaria. Around $10 \%$ of Omani nationals are carriers of the allele for sickle cell anemia, 2-3\% carry an allele for Beta-thalassemia and $45 \%$ are carriers of an alpha-thalassemia allele ${ }^{12-15}$.

Genetic disorders causing disabilities and handicap are of great concern. These are different groups of rare disorders leading to intellectual disability or physical handicap requiring detailed clinical classification, genetic testing, research and preventive measures. The high prevalence of birth defects and genetic conditions in Omani communities causes social, psychological and financial difficulties ${ }^{4}$. The development and use of national mutation data is of importance to Omani medical care because it not only allows the genetic burden of disease to be quantified, but also provides diagnosticians and researchers access to an up-to-date resource that will assist them in their daily clinical practice and biomedical research ${ }^{9}$. National databases for genetic variants are also significant from the perspective of preventive healthcare. There is a significant correlation between the occurrence of rare genetic variants associated with Mendelian disease and the burden of morbidity from complex diseases within a population. Heterozygous carriers for recessive disease genes do not manifest the recessive disease but may be at risk of developing complex trait conditions with some similarity in phenotype. For example, heterozygote carriers of mutations in the ataxia telangiectasia gene locus are reportedly susceptible to breast cancer $^{16}$, and heterozygote carriers of mutations in the glucocerebrosidase (GBA) gene causing Gaucher disease are at an increased risk for Parkinson disease ${ }^{17,18}$. Hence, the collection of genetic variant data in national databases will contribute significantly to the prevention of genetic diseases in the population and might greatly impact the management of complex trait diseases in the future. Genetic scientists and international consortiums studying human genetic variation are increasingly interested in dissecting the interplay between genetic makeup and environmental influences on the pattern of diseases worldwide. Current research is expected to create a foundation for the national data online for the benefit of Oman Healthcare.

\section{Data availability}

This article was prepared to introduce the first Omani genetic variation database. This data is available online at HTTP://ogvd.net; raw datasets are not available for Royal Hospital laboratory and clinical data, as the registry contains confidential information that could not be deidentified.

\section{Author contributions}

RA and MB conceived the study. RA prepared the first draft of the manuscript. RA, AHS, ALF, GU, MH, HS and ASQ carried out the research. $\mathrm{HN}, \mathrm{AI}, \mathrm{KK}, \mathrm{MB}$ and $\mathrm{RP}$ contributed to the preparation of the manuscript. All authors were involved in the revision of the draft.

\section{Competing interests}

The authors declared no competing interests.

\section{Grant information}

The author(s) declared that no funding was involved in supporting this work.
1. Tadmouri GO Al Ali MT, Al Khaja N: Genetic disorders in Arab World: Oman Publication of Center for Arab Genomic Studies, Dubai. 2008; 3. Reference Source

2. Rajab A: Genetic Disorders in Oman. In: Teebi AS (ed): Genetic Disorders Among Arab Populations. Berlin: Springer, 2010; 473-490. Publisher Full Text

3. Rajab A, Patton M: Genetic diseases in the Sultanate of Oman. In: Dhavendra Kumar (ed.), Genomics and health in the developing world; Oxford monograph on medical genetics. Oxford University Press, 2012; 678-693. Publisher Full Text

4. Rajab A, Al Salmi Q, Jaffer J, et al:: Congenital and genetic disorders in the Sultanate of Oman. First attempt to assess healthcare needs. $J$ Community Genet. 2014; 5(3): 283-9.

PubMed Abstract | Publisher Full Text | Free Full Text

5. Rajab A, Bappal B, Al-Shaikh $\mathrm{H}$, et al:: Common autosomal recessive diseases in Oman derived from a hospital-based registry. Community Genet. 2005; 8(1): 27-30. PubMed Abstract | Publisher Full Text

6. Al-Thihli K, Al-Murshedi F, Al-Hashmi N, et al.: Consanguinity, endogamy and inborn errors of metabolism in Oman: a cross-sectional study. Hum Hered. 2014; 77(1-4): 183-8.

PubMed Abstract | Publisher Full Text

7. Rajab A, Patton MA: Analysis of the population structure in Oman. Community Genet. 1999; 2(1): 23-5

PubMed Abstract | Publisher Full Text

8. Rajab A, Al Rashdi I, AI Salmi Q: Genetic services and testing in the Sultanate of Oman. Sultanate of Oman steps into modern genetics. J Community Genet. 2013; 4(3): 391-397.

PubMed Abstract | Publisher Full Text | Free Full Text

9. Patrinos GP, Smith TD, Howard H, et al:: Human Variome Project country nodes: documenting genetic information within a country. Hum Mutat. 2012; 33(11): 1513-9

PubMed Abstract | Publisher Full Text
10. Patrinos GP, Cooper DN, van Mulligen E, et al.: Microattribution and nanopublication as means to incentivize the placement of human genome variation data into the public domain. Hum Mutat. 2012; 33(11): 1503-12. PubMed Abstract | Publisher Full Text

11. Rajab A, Patton MA: Major factors determining the frequencies of hemoglobinopathies in Oman. Letter to the Editor. Am J Med Genet. 1997; 71(2) 240-242.

PubMed Abstract | Publisher Full Text

12. Alkindi S, Al Zadjali S, Al Madhani A, et al:: Forecasting hemoglobinopathy burden through neonatal screening in Omani neonates. Hemoglobin. 2010; 34(2): 135-44.

PubMed Abstract | Publisher Full Text

13. White JM, Christie BS, Nam D, et al: Frequency and clinical significance of erythrocyte genetic abnormalities in Omanis. J Med Genet. 1993; 30(5): 396-400. PubMed Abstract | Publisher Full Text | Free Full Text

14. Daar S, Hussein HM, Merghoub T, et al.: Spectrum of beta-thalassemia mutations in Oman. Ann NY Acad Sci. 1998: 850: 404-6. PubMed Abstract | Publisher Full Text

15. Daar S, Hussain HM, Gravell D, et al.: Genetic epidemiology of HbS in Oman: multicentric origin for the $\beta^{\mathrm{s}}$ gene. Am J Hematol. 2000; 64(1): 39-46. PubMed Abstract | Publisher Full Tex

16. Athma P, Rappaport R, Swift M: Molecular genotyping shows that ataxiatelangiectasia heterozygotes are predisposed to breast cancer. Cancer Genet Cytogenet. 1996; 92(2): 130-4. PubMed Abstract | Publisher Full Text

17. Goker-Alpan O, Schiffmann R, LaMarca ME, et al:: Parkinsonism among Gaucher disease carriers. J Med Genet. 2004; 41(12): 937-40. PubMed Abstract | Publisher Full Text | Free Full Text

18. Sidransky $\mathrm{E}$, Samaddar $\mathrm{T}$, Tayebi N: Mutations in GBA are associated with familial Parkinson disease susceptibility and age at onset. Neurology. 2009; 73(17): 1424-5, author reply 1425-6. PubMed Abstract | Publisher Full Tex 


\section{Open Peer Review}

\section{Current Peer Review Status:}

\section{Version 1}

Reviewer Report 26 October 2015

https://doi.org/10.5256/f1000research.7471.r10917

(C) 2015 Macek M. This is an open access peer review report distributed under the terms of the Creative Commons Attribution License, which permits unrestricted use, distribution, and reproduction in any medium, provided the original work is properly cited.

\section{Milan Macek}

Department of Biology and Medical Sciences, Charles University in Prague, Prague, Czech Republic

This is an excellent pioneering piece of work which presents an authoritative overview of a representative mutation analysis (300 pathogenic variants in over 150 monogenic disorders) in a large cohort of Omani patients. Methodology is state of the art, collaboration with colleagues at Charite Berlin commendable and results which are important for DNA diagnostics in the Omani population, and beyond. The conclusions, are relevant and directly applicable. I fully approve this article without any comments to the Authors. Importantly, data are also freely accessible online.

Competing Interests: No competing interests were disclosed.

\section{I confirm that I have read this submission and believe that I have an appropriate level of expertise to confirm that it is of an acceptable scientific standard.}

Reviewer Report 14 October 2015

\section{https://doi.org/10.5256/f1000research.7471.r10729}

(C) 2015 Dogra S. This is an open access peer review report distributed under the terms of the Creative Commons Attribution License, which permits unrestricted use, distribution, and reproduction in any medium, provided the original work is properly cited.

\section{Shaillay Dogra}

Vishuo BioMedical Pte Ltd, Singapore, Singapore

Its useful to have region/country specific databases that present a better representation of the prevalence and incidence of region specific diseases and health issues. Putting such data in context of whats known globally shall help in better interpreting whats peculiar to local population and possibly raise some interesting questions on why so? For example, is it the genetic structure or something in the environment? 
Some specific comments follow:

1. Authors mention existence of tribal structures, consanguineous marriages etc in Omani society. Is it possible to delineate the effects of these factors on the reported mutations? For example, is this reported in the papers that the authors have collated data from and have the authors noted this aspect in their database?

2. How does the mutation data reported here compare with mutation patterns or frequency seen in other populations? Do the authors provide information on this or link out to other similar resources from other countries? If a physician in Oman is looking up some mutation from this database and wants to know if this is something specific to Oman or is a more general mutation found in other populations too, would this information appear automatically in the database; or could they perform a manual search of the database?

3. Do the authors want to comment about any data privacy issues that maybe associated with such a database, if any and to what extent?

4. Are there any attributes on quality of data in the database? perhaps based on the technique used in the original paper or some other measure some quality metric can be assigned to the mutation information recorded in the database?

5. It would be helpful if authors were able to illustrate out a case or two on how they expect a doctor in Oman to be able to use this database in a real clinical setting; to illustrate the usefulness of the database from a simple collection of data to something that can be used on a more regular basis by doctors in clinical setting.

Competing Interests: No competing interests were disclosed.

I confirm that I have read this submission and believe that I have an appropriate level of expertise to confirm that it is of an acceptable scientific standard.

Reviewer Report 09 October 2015

https://doi.org/10.5256/f1000research.7471.r10505

(C) 2015 Ranganath P. This is an open access peer review report distributed under the terms of the Creative Commons Attribution License, which permits unrestricted use, distribution, and reproduction in any medium, provided the original work is properly cited.

\section{Prajnya Ranganath}

Department of Medical Genetics, Nizam's Institute of Medical Sciences, Hyderabad, Telangana, India

1. The paper is well-written and though it does not present any new findings, it can be used as a reference database for Omani mutations.

2. There are a few suggestions for the authors: 
a. Standard HGVS nomenclature should be followed for all the mutations and the authors should preferably stick to one format i.e. either DNA or protein notation. There is no uniformity in the present nomenclature followed in Table 2.

b. Number of patients in whom each mutation was found, should be indicated - this would give an idea about any preponderance of specific mutations in this population.

c. Functional validation studies, if available, or at least the mutation prediction scores should be mentioned for the novel sequence variants identified, which will help create a comprehensive database of new likely-pathogenic variants. Again, if the number of patients in whom each of these novel variants were identified is mentioned, we will get an idea as to whether these novel variants were present in more than one case and we can get further proof of the pathogenicity of these mutations.

d. It would also be interesting to see if there is any ethnic group-wise preponderance of genetic diseases or mutations in the various subsets/ tribes/ regional groups that constitute the Omani population.

Competing Interests: No competing interests were disclosed.

I confirm that I have read this submission and believe that I have an appropriate level of expertise to confirm that it is of an acceptable scientific standard.

\section{Comments on this article}

\section{Version 1}

\section{Reader Comment 08 Nov 2017}

Majid Al Salmani, School of Physiology, Pharmacology and Neuroscience, University of Bristol, UK

First of all, I congratulate you for this excellent piece of work. I would love to see the mutation database initiated as soon as possible, as this will help researchers from Oman and from Other countries too. For this, I would like to see a link to the database website. The article is fantastic. However, it would have been better if you could give references to the published data about the disease causing mutations, particularly those in Table 2. I noticed, for example, that the mutations of the disease cystic fibrosis (Table 2) had inconsistencies when compared with published data. First, the mutation F208Del does not exist, because there is no amino acid as F208 in CFTR. You might have meant F508del, but you have mentioned this already as Delta F508. Second, the mutation S549Rdel does not exist in literature, and the nomenclature (mutation name) might be wrong!! Third, there are few additional CFTR mutations that have been reported in Omani patients, but have not been mentioned in this table. Thank you very much.

Competing Interests: There are no competing interests 
The benefits of publishing with F1000Research:

- Your article is published within days, with no editorial bias

- You can publish traditional articles, null/negative results, case reports, data notes and more

- The peer review process is transparent and collaborative

- Your article is indexed in PubMed after passing peer review

- Dedicated customer support at every stage

For pre-submission enquiries, contact research@f1000.com 